\title{
The efficacy and safety of abiraterone acetate in patients with high-risk prostate cancer: a meta-analysis based on six randomized control trials
}

\author{
Guobin Tan ${ }^{1 \#} \wedge$, Zijun Xuan ${ }^{2 \#}$, Zhiqin $\mathrm{Li}^{1}$, Shuitong Huang ${ }^{1}$, Guangming Chen ${ }^{1}$, Yonglu Wu ${ }^{1}$, Xianxi Chen ${ }^{1}$, \\ Zhijin Liang', Aiming $\mathrm{Wu}^{1}$ \\ ${ }^{1}$ Department of Urology, Maoming People's Hospital, Maoming, China; ${ }^{2}$ Department of Urology, Dongguan Kanghua Hospital, Dongguan, China \\ Contributions: (I) Conception and design: G Tan; (II) Administrative support: A Wu; (III) Provision of study materials or patients: A Wu; (IV) \\ Collection and assembly of data: G Tan, Z Li, S Huang, G Chen, Y Wu, X Chen, Z Liang; (V) Data analysis and interpretation: G Tan, Z Xuan; (VI) \\ Manuscript writing: All authors; (VII) Final approval of manuscript: All authors. \\ "The authors contributed equally to this work. \\ Correspondence to: Aiming Wu. Department of Urology, Maoming People’s Hospital, Maoming, China. Email: 18666818362@163.com.
}

\begin{abstract}
Background: Abiraterone acetate, a CYP17 enzyme inhibitor, can block the synthesis of androgens in the adrenal gland, prostate, and testis. The purpose of this study was to investigate the efficacy and safety of abiraterone acetate in high-risk prostate cancer patients, including metastatic castration-resistant prostate cancer (mCRPC) and metastatic castration-sensitive prostate cancer (mCSPC).

Methods: A meta-analysis based on 6 randomized controlled trials (RCTs) was undertaken in compliance with the guidelines of systematic reviews and meta-analyses. Databases including PubMed, EMBASE, and Cochrane library were searched for relevant literature through to September 2019.

Results: The pooled analysis reported abiraterone acetate showed significant efficacy in high-risk prostate cancer patients, including overall survival (OS) [HR 0.66, 95\% confidence interval (CI), 0.61-0.73, $\mathrm{P}<0.001$, the time to prostate-specific antigen (PSA) progression (HR 0.45, 95\% CI, 0.34-0.59, $\mathrm{P}<0.001$ ), progressionfree survival (PFS) (according to radiographic evidence) (HR 0.55, 95\% CI, 0.45-0.68, P<0.001) and PSA response rate (RR 2.49, 95\% CI, 1.47-4.22, $\mathrm{P}<0.001)$. A subgroup analysis was carried out due to the significant heterogeneity between the studies. The incidence of arthralgia (RR 1.19), hypokalemia (RR 2.47), cardiac disorder (RR 1.48), and hypertension (RR 1.57) in the abiraterone acetate group was moderately higher than the control group.
\end{abstract}

Conclusions: The efficacy and safety of abiraterone acetate as an androgen receptor (AR) pathway targeted drug in high-risk prostate cancer patients was confirmed.

Keywords: Abiraterone acetate; prostate cancer; efficacy; safety

Submitted Jun 09, 2020. Accepted for publication Aug 05, 2020.

doi: $10.21037 /$ tau-20-1058

View this article at: http://dx.doi.org/10.21037/tau-20-1058

\section{Introduction}

In Europe and America, prostate cancer is the most common urogenital cancer (1). In America, about $3 \%$ of prostate cancer patients are diagnosed with metastatic castration-sensitive prostate cancer (mCSPC). The conventional treatment for $\mathrm{mCSPC}$ is androgen deprivation therapy (ADT), which includes luteinizing hormonereleasing hormone analogs, bilateral testicular resection, or first-generation androgen receptor (AR) blocker. Also, this standard treatment strategy has been proposed for

^ ORCID: 0000-0003-1813-371X. 
this kind of patient for a long time (2). Although most patients have an initial response to ADT, within about a year, the majority progress to become castration-resistant prostate cancer (CRPC) $(3,4)$. AR signaling promotes CRPC primarily through up-regulation of testosterone production in tumors, sustained production of adrenal androgen, parallel pathways of steroid production, and changes to biological characteristics of ARs (2). New endocrine drugs for the treatment of CRPC include enzalutamind and abiraterone acetate. In addition, cytotoxic drugs and immunotherapeutic drugs such as docetaxel, cabazitaxel, mitoxantrone, estramustine, and Sipuleucel-T are also used to treat CRPC. Abiraterone acetate, a CYP17 enzyme inhibitor, can block the synthesis of androgen in the adrenal gland, prostate, and testis (5). Several highquality randomized controlled trials (RCTs) have stated that abiraterone acetate plus prednisone (AAP) achieved significant efficacy in high-risk prostate cancer patients, including castration-resistant and castration-sensitive, and also provided additional clinical benefits (6-17). Previous studies (18-20) have also demonstrated the efficacy and safety of abiraterone acetate in high-risk prostate cancer patients. However, the difference in efficacy of abiraterone acetate between metastatic castration-resistant prostate cancer (mCRPC) and mCSPC remains unclear, as does whether earlier use of abiraterone acetate in $\mathrm{mCSPC}$ could improve clinical benefits. In this meta-analysis, we aimed to investigate the efficacy of abiraterone acetate in mCRPC and $\mathrm{mCSPC}$ based on 6 RCTs. We present the following article in accordance with the PRISMA reporting checklist (available at http://dx.doi.org/10.21037/tau-20-1058).

\section{Methods}

\section{Literature search}

We searched PubMed, EMBASE, Cochrane library for literature about the efficacy and safety of abiraterone acetate in high-risk prostate cancer patients (including castrationresistant and castration-sensitive) through to September 2019. The search criteria were limited to articles of Phase III or phase II RCTs. The search was performed using the following search strategy (in PubMed):

* \#1. prostate cancer

* \#2. abiraterone acetate

* \#3. randomized [Title/Abstract] OR randomized controlled trial [Publication Type] OR placebo [Title/ Abstract]
* \#4. \#1 and \#2 and \#3

Two researchers independently reviewed all the literature obtained and selected suitable studies.

\section{Study selection}

All studies meeting the following criteria were included: (I) study design type was phase III or phase II RCT; (II) the patients were diagnosed with high-risk prostate cancer, including castration-resistant and castration-sensitive; (III) abiraterone acetate was set as the experimental drug; (IV) a control arm as comparison was available; (V) overall survival (OS), the time to prostate-specific antigen (PSA) progression, and progression-free survival (PFS) (according to radiographic evidence) was expressed as a hazard ratio (HR), while PSA response rate and relative adverse events were expressed as risk ratios (RR); (VI) the language of the study was English. Exclusion criteria included nonRCTs, studies with insufficient data, systematic reviews, case reports, and duplicate studies. All the eligible studies were evaluated by two reviewers independently, according to the main criteria, and disagreements were resolved by consulting with a third researcher.

\section{Data extraction}

The following data for each eligible study, such as the first author's last name, year of publication, site of origin, number of cases and controls, and associated outcomes, were extracted by two reviewers independently. A third reviewer was available for consultation in the instance of disagreement.

\section{Methodological quality assessment}

To assess the quality of included studies, we used the Jadad 5 -item scale, the score of which ranged from 0 to 5 , taking into account randomization, double-blinding, withdrawals, and dropouts (21).

\section{Statistical analysis}

The statistical analyses were performed using RevMan 5.3. A random-effects model (DerSimoniane-Laird method) or a fixed-effects model (Mantel-Haenszel method) was used to generate the summary estimates according to the heterogeneity. Statistical heterogeneity was calculated for included studies by using the chi-squared $\left(\chi^{2}\right)$ and the 


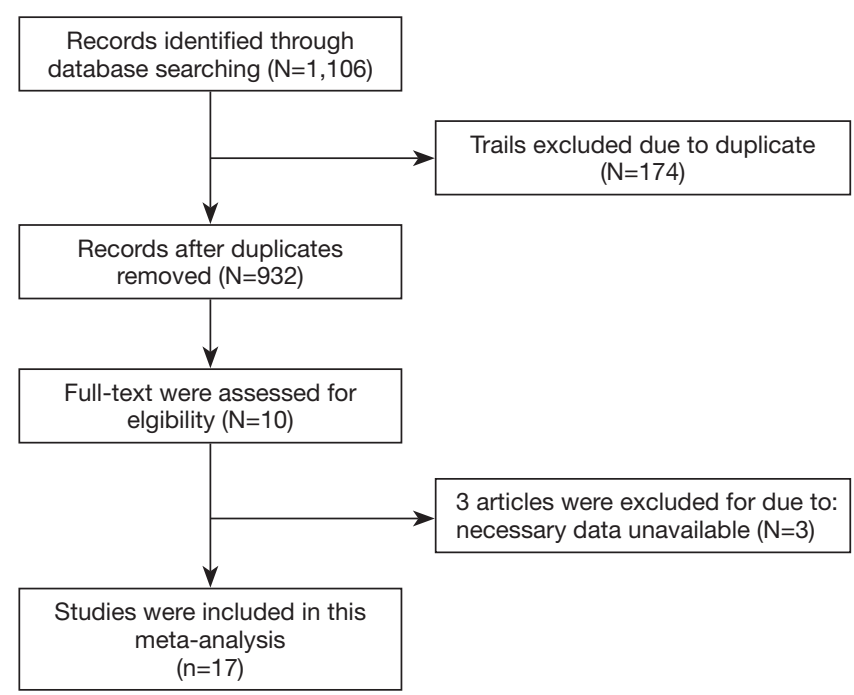

Figure 1 Trial selection flow chart.

heterogeneity coefficient $\left(\mathrm{I}^{2}\right)$ tests. The fixed-effects model was applied when $\mathrm{P}>0.1$ and $\mathrm{I}^{2}<50 \%$; otherwise, the randomeffects model was applied (22). HRs with $95 \%$ confidence intervals (CIs) of OS, the time to PSA progression, and the PFS (according to radiographic evidence), which were timeto-event variables, were calculated for each study. For the dichotomous variables, RRs with $95 \%$ CI of PSA response rate and adverse events were calculated for each study. Furthermore, subgroup analysis was performed to identify any differences for the endpoints between mCRPC and mCSPC. $\mathrm{P}<0.05$ was regarded as statistically significant for all the statistical analyses; all tests were two-sided. Publication bias was not assessed because the number of included studies was less than 10 .

\section{Results}

\section{Identification of eligible studies}

In the initial search, a total of 1,106 studies were identified, 10 studies remained after removing duplicates, case reports, reviews, and unrelated studies through assessing the abstracts. A further review excluded 3 other studies as they were found to be lacking some essential data (Figure 1), leaving 7 studies (11-17) to be included. The whole process was completed by two researchers independently, with differences settled by consulting with a third researcher.

A total of seven studies were included in the metaanalysis. Fizazi (2017) and Fizazi (2019) belonged to the same RCT. However, they had different endpoints. All the included studies were published between 2011 and 2019. A total of 5,901 patients were involved in the meta-analysis, 3,190 cases were treated with AAP or AAP combined with $\mathrm{ADT}$, and 2,711 controls were treated with placebo plus prednisone or ADT alone. Four studies (11,15-17) enrolled 2,810 patients with mCRPC, while the other 3 studies (12-14) enrolled 3,116 patients with mCSPC. The characteristics and quality assessment by the Jadad 5-item scale of the included studies were summarized in Table 1. Of the 7 studies, all except James (2017), were randomized, double-blind clinical trials. In Bono (2011), Ryan (2012), Sun (2016), and Ye (2017), the experimental arm was set as AAP, while the control arm was set as placebo plus prednisone. In James (2017) and Fizazi (2019), the comparison was set as AAP combined with ADT, versus $\mathrm{ADT}$ alone or prednisone plus $\mathrm{ADT}$.

\section{OS}

The pooled analysis for 5 studies $(11,13-16)$ reported abiraterone acetate showed significant improvement of OS in high-risk prostate cancer patients (HR 0.66, 95\% CI, $0.61-0.73, \mathrm{P}<0.001 ; \mathrm{I}^{2}=0 \%$, Figure 2), but no significant heterogeneity was found between $\mathrm{mCRPC}$ and $\mathrm{mCSPC}$ $\left(\mathrm{I}^{2}=0 \%, \mathrm{P}=0.44\right.$, Figure 2$)$ by the subgroup analysis. Due to no significant heterogeneity $\left(\mathrm{I}^{2}=0 \%\right)$ between the trials, a fixed-effects model was used.

\section{The time to PSA progression}

The pooled analysis for 5 studies $(11,13,15-17)$ reported abiraterone acetate showed significant improvement in the time to PSA progression in high-risk prostate cancer patients (HR 0.45, 95\% CI, 0.34-0.59, P $<0.00001$; $\mathrm{I}^{2}=86 \%$, Figure 3), while a significant heterogeneity was found between $\mathrm{mCRPC}$ and $\mathrm{mCSPC}\left(\mathrm{I}^{2}=96.3 \%, \mathrm{P}<0.001\right.$, Figure 3) by the subgroup analysis. Due to the significant heterogeneity between the trials, a random-effects model was used. We found that significant heterogeneity might be caused by Fizazi (2019), which enrolled the patients with mCSPC.

\section{PFS according to radiographic evidence}

The pooled analysis for 3 studies $(11,12,15)$ reported abiraterone acetate showed significant improvement of PFS in high-risk prostate cancer patients (HR 0.55, 95\% CI, 0.45-0.68, $\mathrm{P}<0.00001 ; \mathrm{I}^{2}=81 \%$, Figure 4), while 
Table 1 Studies characteristic

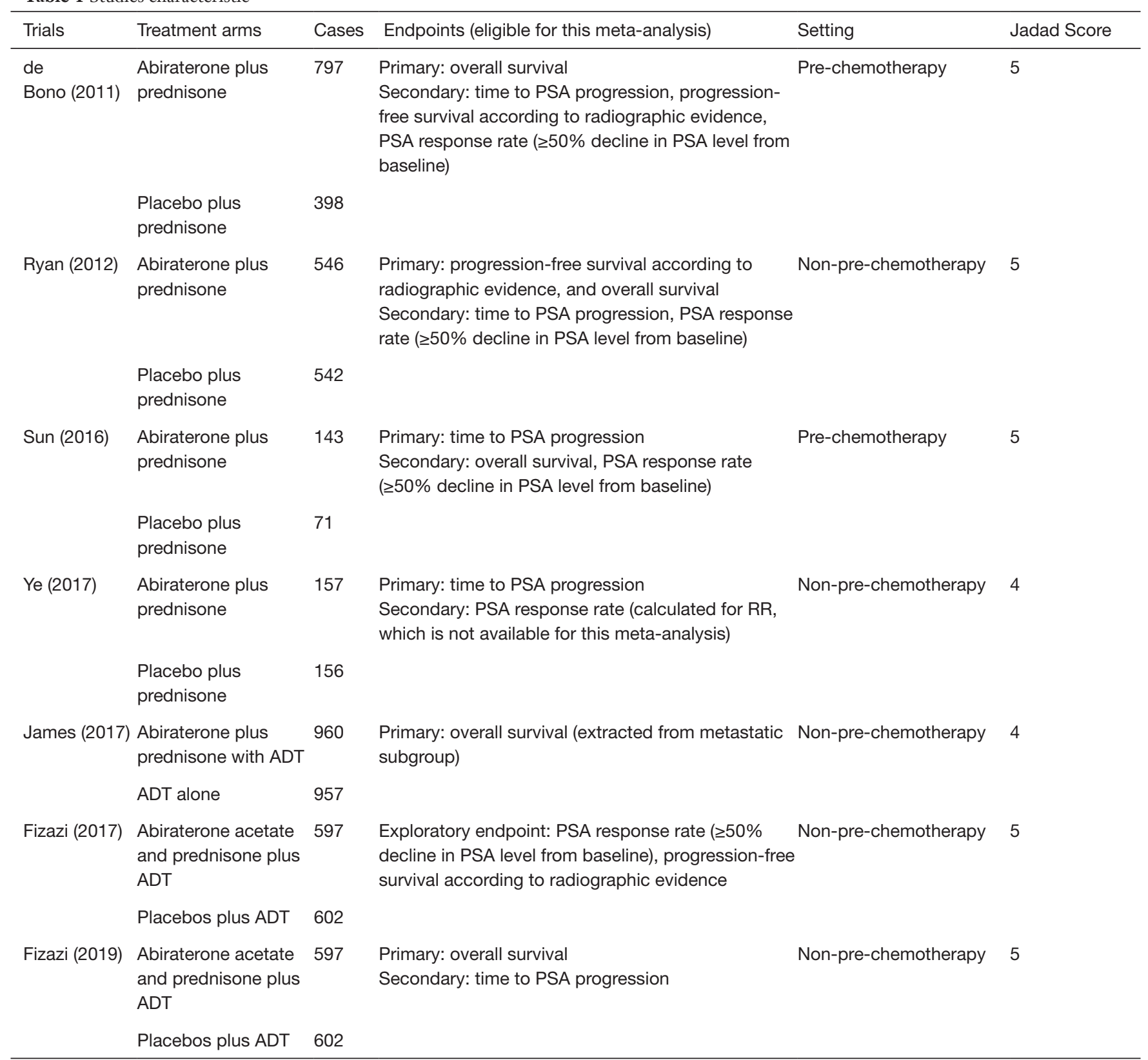

PSA, prostate-specific antigen; ADT, androgen deprivation therapy.

a significant heterogeneity was found between mCRPC and $\mathrm{mCSPC}\left(\mathrm{I}^{2}=63.1 \%, \mathrm{P}=0.10\right.$, Figure 4$)$ by the subgroup analysis. Due to the significant heterogeneity between the trials, a random-effects model was used. However, we cannot remove the heterogeneity within the subgroup. Furthermore, we set the subgroups as pre-chemotherapy and non-pre-chemotherapy (Figure 5) and then observed that the significant heterogeneity might be caused by
Bono (2011), which enrolled patients with mCRPC and pre-chemotherapy.

\section{PSA response rate}

The pooled analysis for 4 studies $(11,12,15,16)$ reported that abiraterone acetate showed significant improvement of PSA response rate in high-risk prostate cancer patients (RR 2.49, 


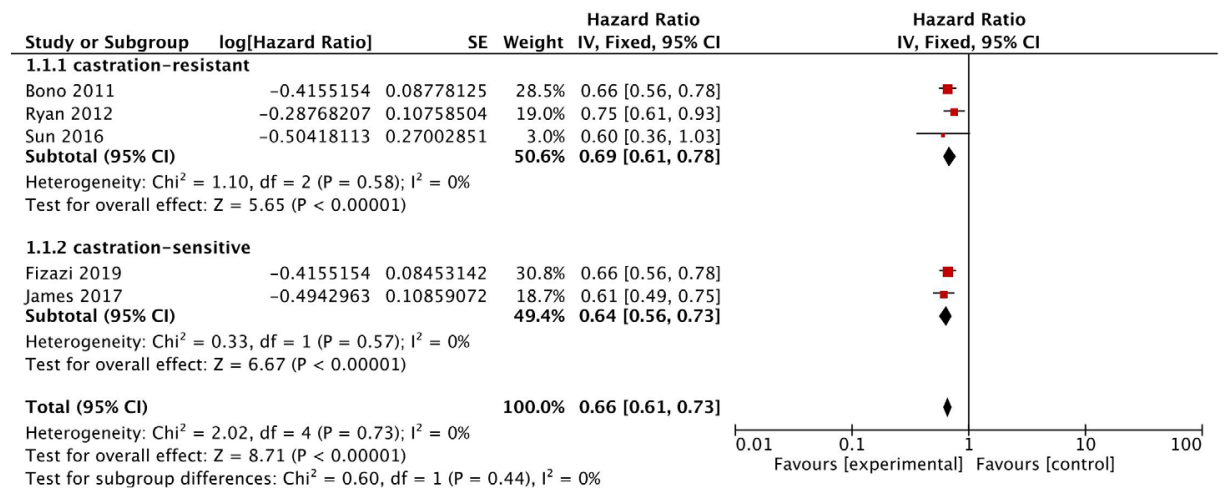

Figure 2 Forest plots of hazard ratios (HRs) for overall survival (OS) comparing experimental arm [abiraterone acetate + prednisone or abiraterone acetate + prednisone + androgen deprivation therapy (ADT)] to the control arm (placebo + prednisone or ADT alone). A subgroup analysis was performed between metastatic castration-resistant prostate cancer and metastatic castration-sensitive prostate cancer. The chi-square test showed no high heterogeneity between the trials. The fixed effects model was used.

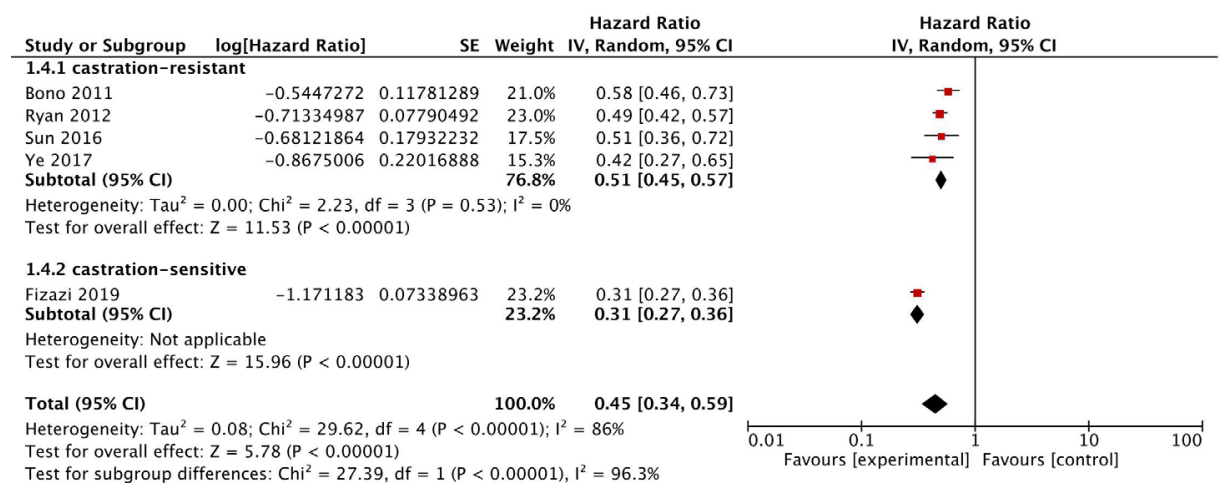

Figure 3 Forest plots of hazard ratios (HRs) for the time to prostate-specific antigen (PSA) progression comparing experimental arm [abiraterone acetate + prednisone or abiraterone acetate + prednisone + androgen deprivation therapy (ADT)] to the control arm (placebo + prednisone or ADT alone). A subgroup analysis was performed between metastatic castration-resistant prostate cancer and metastatic castration-sensitive prostate cancer. The chi-square test showed high heterogeneity between the trials. The random effects model was used.

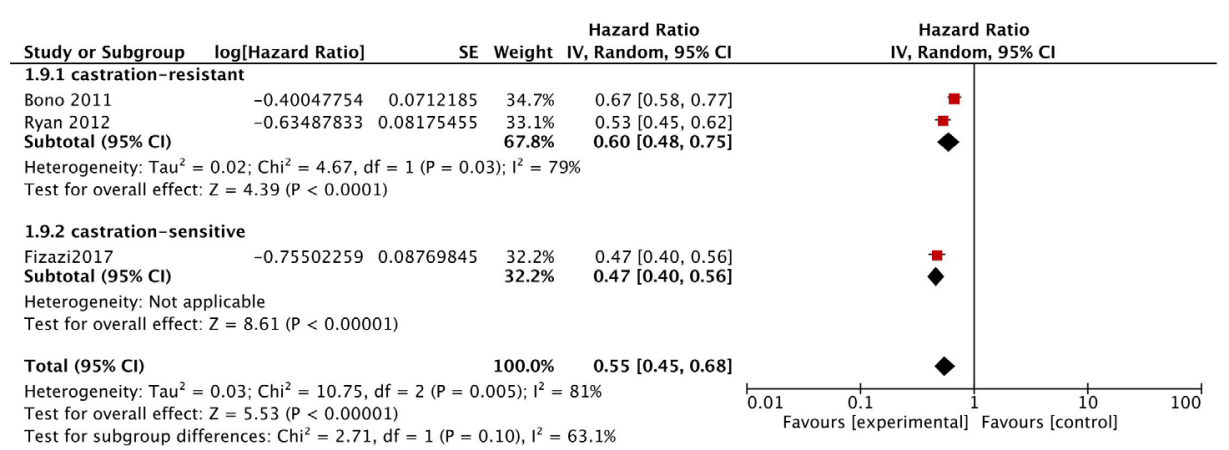

Figure 4 Forest plots of hazard ratios (HRs) for progression-free survival (according to radiographic evidence) comparing experimental arm [abiraterone acetate + prednisone or abiraterone acetate + prednisone + androgen deprivation therapy (ADT)] to the control arm (placebo + prednisone or ADT alone). A subgroup analysis was performed between metastatic castration-resistant prostate cancer and metastatic castration-sensitive prostate cancer. The chi-square test showed high heterogeneity between the trials. The random effects model was used. 


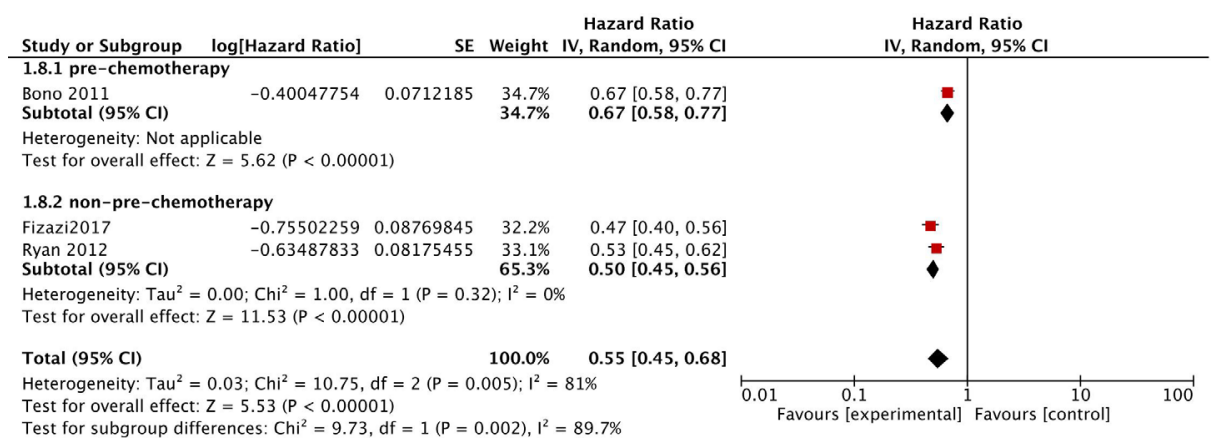

Figure 5 Forest plots of hazard ratios (HRs) for progression-free survival (according to radiographic evidence) comparing experimental arm [abiraterone acetate + prednisone or abiraterone acetate + prednisone + androgen deprivation therapy (ADT)] to the control arm (placebo + prednisone or ADT alone). A subgroup analysis was performed between pre-chemotherapy and non-pre-chemotherapy. The chi-square test showed high heterogeneity between the trials. The random effects model was used.

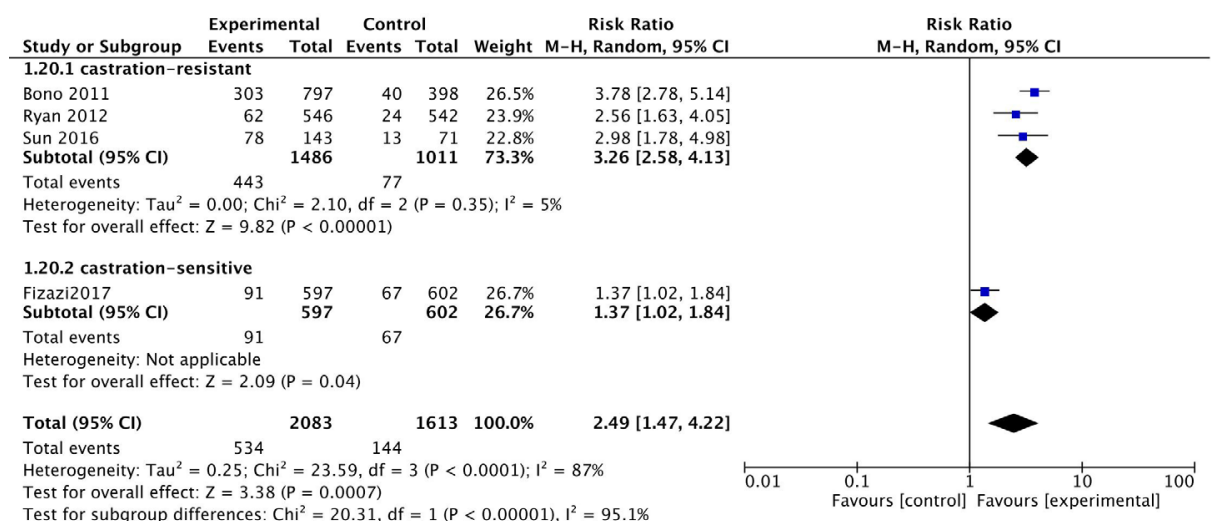

Figure 6 Forest plots of risk ratios (RRs) for prostate-specific antigen (PSA) response rate comparing experimental arm [abiraterone acetate + prednisone or abiraterone acetate + prednisone + androgen deprivation therapy (ADT)] to the control arm (placebo + prednisone or ADT alone). A subgroup analysis was performed between metastatic castration-resistant prostate cancer and metastatic castration-sensitive prostate cancer. The chi-square test showed high heterogeneity between the trials. The random effects model was used.

95\% CI, 1.47-4.22, P=0.0007; $\mathrm{I}^{2}=87 \%$, Figure 6), while a significant heterogeneity had been found between $\mathrm{mCRPC}$ and $\mathrm{mCSPC}\left(\mathrm{I}^{2}=95.1 \%, \mathrm{P}<0.00001\right.$, Figure 6$)$ in subgroup analysis. Due to the significant heterogeneity between the trials, a random-effects model was used. We found that significant heterogeneity might be caused by Fizazi (2017), which enrolled patients with mCSPC.

\section{Adverse events}

The original data of adverse events in all grades were extracted from the eligible studies and calculated for RRs. In this meta-analysis, adverse events included asthenia, fatigue, back pain, constipation, arthralgia, bone pain, hypokalemia, cardiac disorder, and hypertension. The pooled analysis reported that abiraterone acetate showed a higher incidence of some adverse events in high-risk prostate cancer patients, including hypokalemia (RR 2.47, 95\% CI, 1.39-4.39, $\mathrm{P}=0.002 ; \mathrm{I}^{2}=86 \%$, Figure S1), hypertension (RR 1.57, 95\% CI, 1.37-1.79, $\mathrm{P}<0.00001 ; \mathrm{I}^{2}=24 \%$, Figure S2), cardiac disorder (RR 1.48, 95\% CI, 1.03-2.13, P=0.04; $\mathrm{I}^{2}=75 \%$, Figure S3) and arthralgia (RR 1.19, 95\% CI, 1.05-1.35, $\mathrm{P}=0.007 ; \mathrm{I}^{2}=0 \%$, Figure S4), but not for asthenia, fatigue, constipation, back pain, and bone pain.

\section{Discussion}

Our meta-analysis of 6 RCTs aimed to investigate the 
efficacy and safety of abiraterone acetate in high-risk prostate cancer patients and to explore any different benefits achieved between MCRPC and MCSPC. To the best of our knowledge, this study was the first to investigate these elements, and to use subgroup analysis to explore the different benefits that were identified. The results demonstrated the efficacy and safety of abiraterone acetate as an AR pathway targeted drug and showed different benefits between patients with $\mathrm{mCRPC}$ and mCSPC, except for OS. This finding is significant because it provides substantial evidence to support clinical research in high-risk prostate cancer patients. The data of the 6 RCTs included in the meta-analysis were very clear and highly consistent, providing accurate estimates. All included RCTs (11,13-17) confirmed that abiraterone acetate achieved a significant and different degree of clinical benefits in OS, the time to PSA progression, PFS (according to radiographic evidence), and PSA response rate in high-risk prostate cancer patients (including mCRPC and mCSPC). Also, several articles $(18,23,24)$ based on meta-analysis paid attention to the comparison of the efficacy of different androgen pathway targeted drugs in high-risk prostate cancer patients. However, no relevant, high-quality RCTs have been conducted to directly compare the efficacy of abiraterone acetate between patients with either mCRPC or mCSPC. In the present study, it was meaningful and interesting for us to identify whether abiraterone acetate achieved any different benefits between MCRPC and mCSPC by subgroup analysis.

The results presented here revealed that abiraterone acetate achieved a better OS (34\%), the time to PSA progression (55\%), PFS (according to radiographic evidence) $(45 \%)$, and the PSA response rate $(249 \%)$ in high-risk prostate cancer patients. Subgroup analysis was performed to find out the source of significant heterogeneity among the studies, when we combined the data of the time to PSA progression, PFS (according to radiographic evidence) and the PSA response rate. According to the results of the subgroup analysis, we found the significant heterogeneity might be caused by abiraterone acetate achieving different benefits at different stages of prostate cancer. As we know, the time to PSA progression, PFS (according to radiographic evidence), and the PSA response rate are the prognostic and predictive factors in highrisk prostate cancer patients $(25,26)$. Abiraterone acetate taken together may achieve different degrees of clinical benefits between $\mathrm{mCRPC}$ and $\mathrm{mCSPC}$, which warrants consideration of pursuing further research comparing the efficacy of abiraterone acetate in patients between mCRPC and $\mathrm{mCSPC}$.

In our study, we observed the potential increase in the incidence of adverse events with the use of abiraterone acetate, mainly grade 1-2 adverse events. However, these adverse events have limited impact on the drug withdrawal rate and dose reduction rate. The mechanism of these adverse events may be related to the blockade of CYP17. The pooled analysis revealed that the incidence of arthralgia (RR 1.19), hypokalemia (RR 2.47), cardiac disorder (RR 1.48), and hypertension (RR 1.57) in the abiraterone acetate group was moderately higher than the control group. At the same time, no statistical difference was found for the other adverse events. Hypokalemia was found to be more likely to occur than the other adverse events. In line with previously published studies $(19,24)$, our study showed that cardiac disorders and hypertension should be paid more attention to follow-up.

This meta-analysis has several limitations. Only 6 RCTs were included, and some estimates of the endpoints presented high heterogeneity among the studies. In the studies included, the primary endpoints were not entirely consistent. James (2017) enrolled patients with nonmetastatic prostate cancer, while the other studies only permitted metastatic prostate cancer patients. It should be noted that 2 studies set AAP plus ADT as the experimental group, while ADT alone was set as the control group. Also, Fizazi (2017) and Fizazi (2019) belong to the same RCT, which investigated different endpoints. The studies included did not register all adverse events, and our analysis was only on those with high incidence.

Presently, the efficacy and safety of abiraterone acetate as an AR pathway targeted drug for high-risk prostate cancer patients is confirmed. More high-quality clinical trials are needed to identify any different benefits of abiraterone acetate for patients between $\mathrm{mCRPC}$ and $\mathrm{mCSPC}$.

\section{Acknowledgments}

Funding: This study is funded by the High-level Hospital Construction Research Project of Maoming People's Hospital, Guangdong Medical Science and Technology Research Fund (No. 2017101819316701), and Maoming Science and Technology Project (NO. 180324131700491).

\section{Footnote}

Reporting Checklist: The authors have completed the 
PRISMA reporting checklist. Available at http://dx.doi. org/10.21037/tau-20-1058

Conflicts of Interest: All authors have completed the ICMJE uniform disclosure form (available at http://dx.doi. org/10.21037/tau-20-1058). The authors have no conflicts of interest to declare.

Ethical Statement: The authors are accountable for all aspects of the work in ensuring that questions related to the accuracy or integrity of any part of the work are appropriately investigated and resolved.

Open Access Statement: This is an Open Access article distributed in accordance with the Creative Commons Attribution-NonCommercial-NoDerivs 4.0 International License (CC BY-NC-ND 4.0), which permits the noncommercial replication and distribution of the article with the strict proviso that no changes or edits are made and the original work is properly cited (including links to both the formal publication through the relevant DOI and the license). See: https://creativecommons.org/licenses/by-nc-nd/4.0/.

\section{References}

1. Siegel RL, Miller KD, Jemal A. Cancer statistics, 2016. CA Cancer J Clin 2016;66:7-30.

2. Spina CS. Androgen deprivation therapy and radiation therapy for prostate cancer: the mechanism underlying therapeutic synergy. Transl Cancer Res 2018;7:S695-703.

3. Gravis G, Boher JM, Joly F, et al. Androgen Deprivation Therapy (ADT) Plus Docetaxel Versus ADT Alone in Metastatic Non castrate Prostate Cancer: Impact of Metastatic Burden and Long-term Survival Analysis of the Randomized Phase 3 GETUG-AFU15 Trial. Eur Urol 2016;70:256-62.

4. Sweeney CJ, Chen YH, Carducci M, et al. Chemohormonal Therapy in Metastatic Hormone-Sensitive Prostate Cancer. N Engl J Med 2015;373:737-46.

5. Carbone M, Yang H, Pass HI, et al. BAP1 and cancer. Nat Rev Cancer 2013;13:153-9.

6. Fizazi K, Scher HI, Molina A, et al. Abiraterone acetate for treatment of metastatic castration-resistant prostate cancer: final overall survival analysis of the COU-AA-301 randomised, double-blind, placebo-controlled phase 3 study. Lancet Oncol 2012;13:983-92.

7. de Bono JS, Logothetis CJ, Molina A, et al. Abiraterone and increased survival in metastatic prostate cancer. $\mathrm{N}$
Engl J Med 2011;364:1995-2005.

8. Rathkopf DE, Smith MR, de Bono JS, et al. Updated interim efficacy analysis and long-term safety of abiraterone acetate in metastatic castration-resistant prostate cancer patients without prior chemotherapy (COU-AA-302). Eur Urol 2014;66:815-25.

9. Ryan CJ, Smith MR, de Bono JS, et al. Abiraterone in metastatic prostate cancer without previous chemotherapy. N Engl J Med 2013;368:138-48.

10. Ryan CJ, Smith MR, Fizazi K, et al. Abiraterone acetate plus prednisone versus placebo plus prednisone in chemotherapy-naive men with metastatic castrationresistant prostate cancer (COU-AA-302): final overall survival analysis of a randomised, double-blind, placebocontrolled phase 3 study. Lancet Oncol 2015;16:152-60.

11. de Bono JS, Logothetis CJ, Molina A, et al. Abiraterone and increased survival in metastatic prostate cancer. $\mathrm{N}$ Engl J Med 2011;364:1995-2005.

12. Fizazi K, Tran N, Fein L, et al. Abiraterone plus Prednisone in Metastatic, Castration-Sensitive Prostate Cancer. N Engl J Med 2017;377:352-60.

13. Fizazi K, Tran N, Fein L, et al. Abiraterone acetate plus prednisone in patients with newly diagnosed highrisk metastatic castration-sensitive prostate cancer (LATITUDE): final overall survival analysis of a randomised, double-blind, phase 3 trial. Lancet Oncol 2019;20:686-700.

14. Antonelli A, Palumbo C, Berruti A. Neoadjuvant androgen deprivation therapy through intense inhibition of the androgen target: "Midsummer Night's Dream" or "Much Ado About Nothing"? Ann Transl Med 2019;7:S230.

15. Ryan CJ, Smith MR, de Bono JS, et al. Abiraterone in metastatic prostate cancer without previous chemotherapy. N Engl J Med 2013;368:138-48.

16. Sun $Y, Z$ Zou Q, Sun Z, et al. Abiraterone acetate for metastatic castration-resistant prostate cancer after docetaxel failure: A randomized, double-blind, placebo-controlled phase 3 bridging study. Int J Urol 2016;23:404-11.

17. Ye D, Huang Y, Zhou F, et al. A phase 3, double-blind, randomized placebo-controlled efficacy and safety study of abiraterone acetate in chemotherapy-naïve patients with mCRPC in China, Malaysia, Thailand and Russia. Asian J Urol 2017;4:75-85.

18. Kang M, Jeong CW, Kwak C, et al. Comparing the clinical efficacy of abiraterone acetate, enzalutamide, and orteronel in patients with metastatic castration-resistant prostate cancer by performing a network meta-analysis of eight randomized controlled trials. Oncotarget 2017;8:59690-7. 
19. Roviello G, Sigala S, Sandhu S, et al. Role of the novel generation of androgen receptor pathway targeted agents in the management of castration-resistant prostate cancer: A literature based meta-analysis of randomized trials. Eur J Cancer 2016;61:111-21.

20. Sathianathen NJ, Koschel S, Thangasamy IA, et al. Indirect Comparisons of Efficacy between Combination Approaches in Metastatic Hormone-sensitive Prostate Cancer: A Systematic Review and Network Meta-analysis. Eur Urol 2020;77:365-72.

21. Jadad AR, Moore RA, Carroll D, et al. Assessing the quality of reports of randomized clinical trials: is blinding necessary? Control Clin Trials 1996;17:1-12.

22. DerSimonian R, Laird N. Meta-analysis in clinical trials. Control Clin Trials 1986;7:177-88.

23. Roviello G, Gatta Michelet MR, D'Angelo A, et al. Role of novel hormonal therapies in the management of nonmetastatic castration-resistant prostate cancer: a literaturebased meta-analysis of randomized trials. Clin Transl

Cite this article as: Tan G, Xuan Z, Li Z, Huang S, Chen G, Wu Y, Chen X, Liang Z, Wu A. The efficacy and safety of abiraterone acetate in patients with high-risk prostate cancer: a meta-analysis based on six randomized control trials. Transl Androl Urol 2020;9(4):1691-1699. doi: 10.21037/tau-20-1058
Oncol 2020;22:1033-9.

24. Roviello G, Sigala S, Sandhu S, et al. Role of the novel generation of androgen receptor pathway targeted agents in the management of castration-resistant prostate cancer: A literature based meta-analysis of randomized trials. Eur J Cancer 2016;61:111-21.

25. Bamias A, Bozas G, Antoniou N, et al. Prognostic and predictive factors in patients with androgen-independent prostate cancer treated with docetaxel and estramustine: a single institution experience. Eur Urol 2008;53:323-31.

26. Beer TM, Hotte SJ, Saad F, et al. Custirsen (OGX011) combined with cabazitaxel and prednisone versus cabazitaxel and prednisone alone in patients with metastatic castration-resistant prostate cancer previously treated with docetaxel (AFFINITY): a randomised, open-label, international, phase 3 trial. Lancet Oncol 2017;18:1532-42.

(English Language Editors: J. Jones and J. Chapnick) 


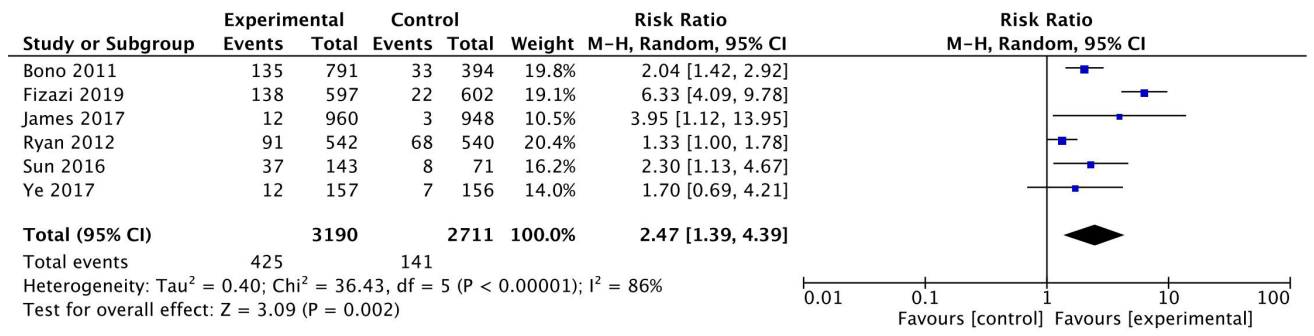

Figure S1 Forest plots of risk ratios (RR) for hypokalemia comparing experimental arm [abiraterone acetate + prednisone or abiraterone acetate + prednisone + androgen deprivation therapy (ADT)] to the control arm (placebo + prednisone or ADT alone). The chi-square test showed significant heterogeneity between the trials. The random effects model was used.

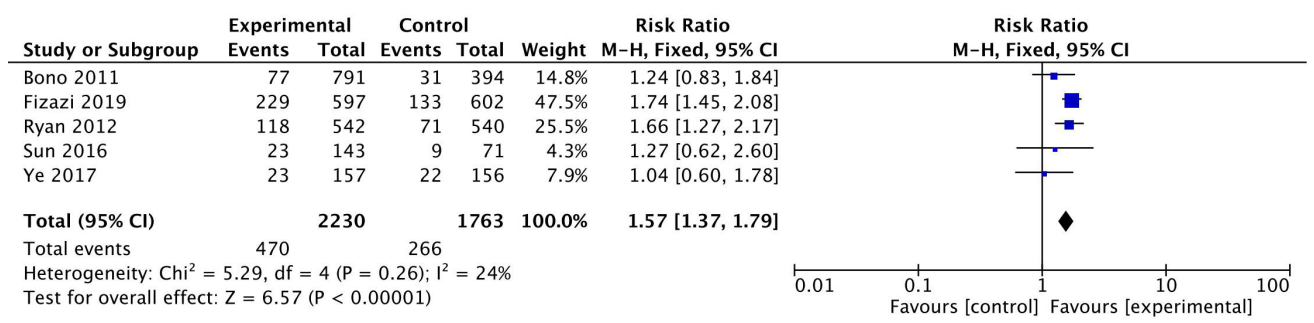

Figure S2 Forest plots of risk ratios (RR) for hypertension comparing experimental arm [abiraterone acetate + prednisone or abiraterone acetate + prednisone + androgen deprivation therapy (ADT)] to the control arm (placebo + prednisone or ADT alone). The chi-square test showed no significant heterogeneity between the trials. The fixed effects model was used.

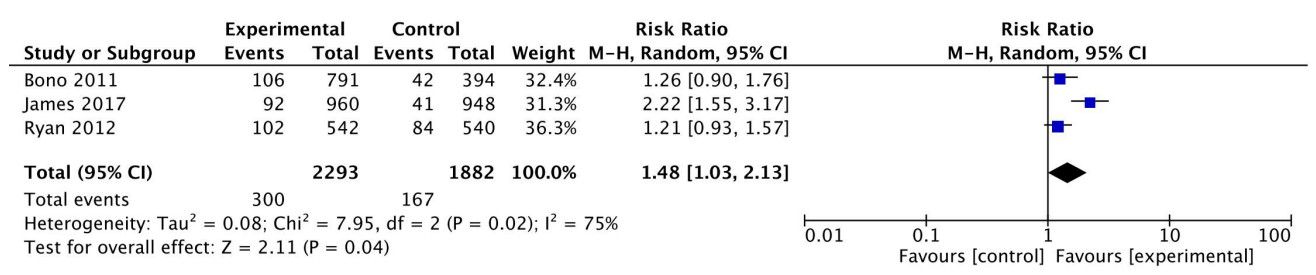

Figure S3 Forest plots of risk ratios (RR) for cardiac disorder comparing experimental arm [abiraterone acetate + prednisone or abiraterone acetate + prednisone + androgen deprivation therapy (ADT)] to the control arm (placebo + prednisone or ADT alone). The chi-square test showed significant heterogeneity between the trials. The random effects model was used.

\begin{tabular}{|c|c|c|c|c|c|c|c|c|c|c|}
\hline Study or Subgroup & \multicolumn{2}{|c|}{ Experimental } & \multicolumn{2}{|c|}{ Control } & Weight & $\begin{array}{c}\text { Risk Ratio } \\
\text { M-H, Fixed, } 95 \% \mathrm{Cl}\end{array}$ & \multicolumn{4}{|c|}{$\begin{array}{c}\text { Risk Ratio } \\
\text { M-H, Fixed, 95\% Cl }\end{array}$} \\
\hline Bono 2011 & 215 & 791 & 89 & 394 & $33.6 \%$ & $1.20[0.97,1.49]$ & & & $E$ & \\
\hline Fizazi 2019 & 96 & 597 & 86 & 602 & $24.2 \%$ & $1.13[0.86,1.47]$ & & & \pm & \\
\hline Ryan 2012 & 154 & 542 & 129 & 540 & $36.6 \%$ & $1.19[0.97,1.45]$ & & & $=$ & \\
\hline Sun 2016 & 17 & 143 & 5 & 71 & $1.9 \%$ & $1.69[0.65,4.39]$ & & & & \\
\hline Ye 2017 & 16 & 157 & 13 & 156 & $3.7 \%$ & $1.22[0.61,2.46]$ & & 7 & $\cdot$ & \\
\hline Total $(95 \% \mathrm{Cl})$ & & 2230 & & 1763 & $100.0 \%$ & $1.19[1.05,1.35]$ & & & $\downarrow$ & \\
\hline Total events & $\begin{array}{r}498 \\
0\end{array}$ & & 322 & & & & & & & \\
\hline $\begin{array}{l}\text { Heterogeneity: } \mathrm{Chi}^{2} \\
\text { Test for overall effec }\end{array}$ & $\begin{array}{l}0.70, \mathrm{df} \\
\mathrm{Z}=2.70\end{array}$ & $\begin{array}{l}=4(P= \\
(P=0.0\end{array}$ & $\begin{array}{l}0.95) ; 1^{2} \\
07)\end{array}$ & $=0 \%$ & & & 0.01 & $\begin{array}{c}0.1 \\
\text { Favours [control] }\end{array}$ & 1 Favours [ex & $\begin{array}{l}10 \\
10 \quad 100 \\
\text { xperimental] }\end{array}$ \\
\hline
\end{tabular}

Figure S4 Forest plots of risk ratios (RR) for arthralgia comparing experimental arm [abiraterone acetate + prednisone or abiraterone acetate + prednisone + androgen deprivation therapy (ADT)] to the control arm (placebo + prednisone or ADT alone). The chi-square test showed no significant heterogeneity between the trials. The fixed effects model was used. 